Non-viral cancer gene therapy

\section{Beyond delivery}

S Akhtar

Gene Therapy (2006) 13, 739-740. doi:10.1038/sj.gt.3302692;

published online 1 December 2005

Although synthetic vectors have generally been considered to be less efficient than their viral counterparts, a recent study suggests that the intrinsic anti-tumour activity of some cationic polymers together with their transfection capability can be exploited to markedly improve the success of cancer gene therapy. ${ }^{1}$ This work could potentially revitalize development of synthetic vectors for gene therapy not only with a view to improving cellular delivery of genes but also to exploit the vector's intrinsic biological activities for additive or synergistic therapeutic effects.

Two of the main reasons why gene therapy has not been globally successful in the clinic are the inefficient delivery of nucleic acids to their correct intracellular sites of action and the poor toxicological profile of some viral-based vectors. Problems such as adenoviral stimulation of the immune response have hastened the search for safer, non-viral vectors for clinical gene therapy. Indeed several different types of cationic synthetic vectors have been investigated for gene therapy applications including lipids/liposomes, polymers, dendrimers (branched-like polymer structures) and cell-penetrating peptides (Figure 1a). ${ }^{2,3}$

The complexes that synthetic vectors form with DNA are often specifically referred to as lipoplexes, dendriplexes or polyplexes depending on whether the vector used is a cationic lipid, dendrimer or polymer, respectively. A vesicular transport system (endocytosis/macropinocytosis) generally takes these complexes into the cell and they are subsequently transported into the nucleus where the delivered gene is expressed.

Previously synthetic vectors have mainly been used for their ability to enhance cellular delivery of nucleic acids. However, in the new work by Christine Dufes et al.,1 published in

b of gene therapy.
Cancer Research, the authors found that the polypropylenimine (PPI) dendrimer vector they used not only had the ability to deliver genes to tumours but also had a modest antitumour activity of its own in xenografts of A431 epidermoid carcinoma or LS174T colorectal adenocarcinoma in nude mice. When they complexed a plasmid that encoded a gene for the tumour necrosis factor-alpha (TNF- $\alpha$ ) (that induces necrotic cell death within solid tumours) to the dendrimer and administered it systemically via tail vein injection to established murine xenografts, they observed a significantly enhanced anti-tumour effect greater than either the dendrimer or plasmid alone.

Remarkably, the enhanced effects on tumour regression led to longterm survival of up to $100 \%$ of the animals after 12 weeks. The authors also reported that two other commonly used polymer formulations, linear polyethylenimine (PEI) and polyamidoamine dendrimer, exerted modest anti-tumour effects of their own, though not as pronounced as the PPI. ${ }^{1}$ These data pave the way for combinations of such formulations with gene therapies for enhanced cancer gene therapy.

Could this approach of exploiting the pharmacological action of the synthetic polymer together with its delivery enhancing capability (albeit less efficient than viruses) be the breakthrough that will help gene therapy realize its true clinical potential? Here, history offers some words of cautionary wisdom. Actually polycations including PEI and PPI have been known to have intrinisic anti-tumour effects for a long time. ${ }^{4-6}$ Previous data ${ }^{6}$ as well as this new study ${ }^{1}$ indicates that the likely
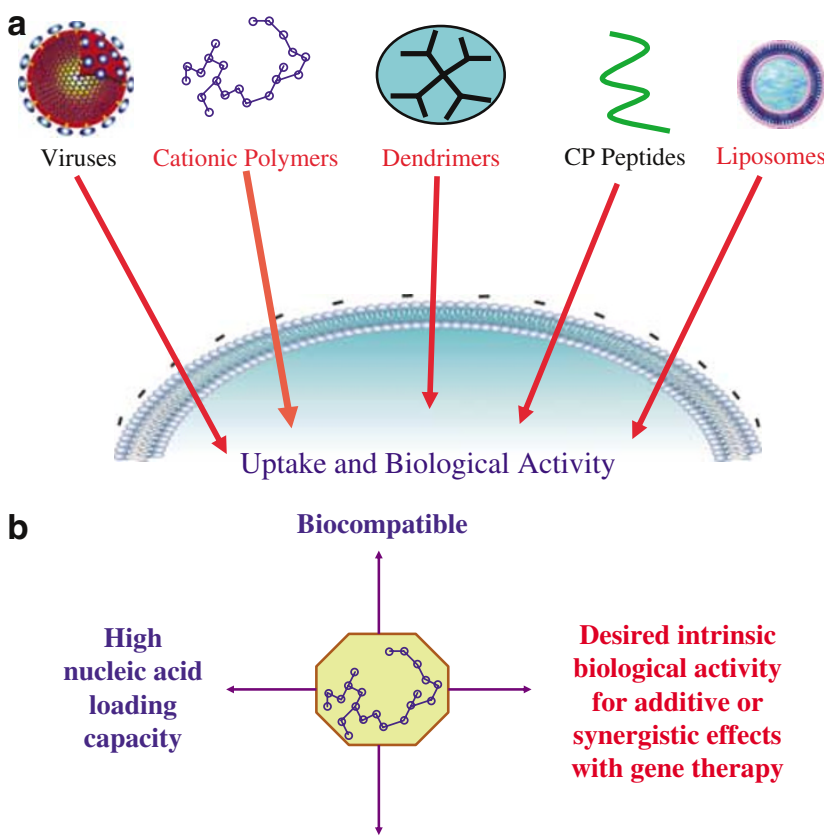

Enhanced

transfection

efficiency

Figure 1 (a) Delivery vectors for intracellular delivery of nucleic acids. Apart from viruses, synthetic cationic vectors such as cationic polymers, branched dendrimers, cell-penetrating (CP) peptides and cationic liposomes can be used to deliver genes into cells. (b) Properties of an engineered synthetic vector for gene therapy in the future. In addition to exhibiting good biocompatibility, loading capacity and transfection efficiency, a future synthetic vector may also be designed to have a desired intrinsic biological activity that would enhance the effects 
mechanism for the polymer-induced anti-tumour activity might be immune stimulation. Moreover, immune modulation may not be peculiar to these cationic polymers because others including polysaccharides such as alginates can also induce TNF- $\alpha$ secretion. ${ }^{7}$

Whereas it seems likely that cationic polymers stimulate the immune system and might also have a direct anti-tumour effect, ${ }^{8,9}$ their exact mechanism of anti-tumour activity remains largely unknown. Recent developments in proteomics and transcriptomics might allow a more detailed insight. Toxicogenomics of non-viral lipidic vectors with microarray-based gene expression profiling has already shown that genes involved in the apoptosis pathway, among others, are altered, by cationic vectors. ${ }^{10}$ More recently, a gene expression profiling study of human A431 cells treated with PPI dendrimers showed that these cationic vectors induce marked global gene expression changes at concentrations routinely used for gene transfection. This finding could potentially indicate that such dendrimers exert pleiotropic biological effects, including induction of apoptosis and some cytokine genes which could be important for their effects on tumour cells. ${ }^{11}$ The extent and type of gene changes elicited appeared to be dependent on the PPI dendrimer generation and cell type, ${ }^{11}$ a finding consistent with those of both Moroson $^{6}$ and Dufes et al. ${ }^{1}$ studies that reported variable anti-tumour activity of the polymers in the different cancer models.

Since Ambrose ${ }^{4}$ and Moroson's ${ }^{5,6}$ early reports, many more polymers (both cationic and anionic) have now been reported to have direct or indirect anti-tumour activity via stimulation of the immune system. These include poly(lysine), poly(arginine), DEAE-Dextrans, polyacrylic acid, several copolymers of maleic anhydride, polysaccharides such as heparin and chitosan. ${ }^{8,9}$ Some polymers showed additive or synergistic anti-tumour effects with conventional cytotoxic agents including enhanced apoptosis ${ }^{12,13}$ and inhibition of multidrug resistance. ${ }^{14}$ Probably, the most clinically successful thus far has been SMANCS, a conjugate of an immunostimulatory styrene-maleic acid copolymer with the anti-tumour polypeptide, neocarzonstatin: this drug reached the market in Japan for use in liver and lung cancer therapy. ${ }^{15}$ However, many cationic polymers have failed to reach the clinic owing to their widespread toxicities. For this reason many of these polymers are now largely considered as potential drug carriers or immunoadjuvants rather than as directly-acting anticancer agents. ${ }^{8}$

It is the previous problems with toxicities of such polymers that make these new findings all the more impressive. The authors not only observed little or no toxicity of their vectors but showed that the combination of the anti-tumour and drug delivery ability of vectors with an appropriate anti-tumour gene therapy leads to remarkable anti-cancer effects in experimental models of solid tumours. A natural extrapolation of this study is that a vector might be engineered to have any desirable property to act in concert with gene therapy for many different pathologies. These results open the door for synthetic chemists to begin engineering new vectors with desirable intrinsic biological activity, for example, anti-tumour activity, improved transfection efficiency and minimal general toxicity (see Figure 1b). If successful, then such designs may have a significant impact on broadening gene therapy applications in the clinic.

S Akhtar is at the Centre for Genome-based Therapeutics, Welsh School of Pharmacy, Cardiff University, Cardiff CF10 3XF, UK.

E-mail:Saghirakhtar@cardiff.ac.uk

Published online 1 December 2005

1 Dufes C, Keith WN, Bilsland A, Proutski I, Uchegbu IF, Schatzlein AG. Synthetic anticancer gene medicine exploits intrinsic antitumor activity of cationic vector to cure established tumors. Cancer Res 2005; 65: 8079-8084.

2 Niidome T, Huang L. Gene therapy progress and prospects: nonviral vectors. Gene Therapy 2002; 24: 1647-1652.

3 Gilmore IR, Fox SP, Hollins AJ, Sohail M, Akhtar S. The design and exogenous delivery of siRNA for post-transcriptional gene silencing. J Drug Targeting 2004; 6: 315-340.
4 Ambrose EJ, Easty DM, Jones PC. Specific reactions of polyelectrolytes with the surfaces of normal and tumour cells. Br J Cancer 1958; 12: 439-447.

5 Furlan M, Moroson H. Cell surface charge increase by both tumour inhibitory and tumour growth promoting polyanions. In: Morosom HL, Quintiliani M (eds). Radiation Protection and Sensitization. Taylor and Francis: London, 1970 (as cited in Mehrishi, JN). Nature 1970; 228: 364-365.

6 Moroson H. Polycation-treated tumor cells in vivo and in vitro. Cancer Res 1971; 31: 373-380.

7 Thomas A, Harding KG, Moore K. Alginates from wound dressings activate human macrophages to secrete tumour necrosis factor. Biomaterials 2000; 21: 1797-1802.

8 Seymour LJ. Synthetic polymers with intrinsic anticancer activity. Bioact Compat Polym 1991; 6: 178-216.

9 Liao J, Ottenbrite RM. Biological effects of polymeric drugs. In: Park K (ed). Controlled Drug Delivery: Challenges and Strategies. American Chemical Society: Washington DC, USA, 1997, pp 455-467.

10 Omidi Y, Hollins AJ, Benboubetra M, Drayton R, Benter IF, Akhtar S. Toxicogenomics of non-viral vectors for gene therapy: a microarray study of lipofectin and oligofectamine-induced gene expression changes in human epithelial cells. J Drug Targeting 2003; 11: 311-323.

11 Omidi Y, Hollins AJ, Drayton RM, Akhtar S. Polypropylenimine dendrimer-induced gene expression changes: the effect of complexation with DNA, dendrimer generation and cell type. $J$ Drug Targeting 2005; 13 (7): 431-443.

12 Minko T, Batrakova EV, Li S, Li Y, Pakunlu RI, Alakhov VY et al. Pluronic block copolymers alter apoptotic signal transduction of doxorubicin in drugresistant cancer cells. J Control Release 2005; 105 (3): 269-278.

13 Minko T, Kopeckova P, Kopecek J. Preliminary evalutation of caspases-dependent apoptosis signaling pathways of free and HPMA copolymer-bound doxrubicin in human ovarian carcinoma cells. J Control Release 2001; 71 (3): 227237.

14 Kabanov AV, Batrakova EV, Sriadibhatla S, Yang Z, Kelly DL, Alakov VY. Polymer genomics: shifting the gene and drug delivery paradigms. I Control Release 2005; 101 (1-3): 259-271.

15 Abe S, Otsuki M. Styrene maleic acid neocarzinostatin treatment for hepatocellular carcinoma. Curr Med Chem AntiCanc Agents 2002; 2 (6): 715-726. 